\title{
Representation of Haltere Oscillations and Integration with Visual Inputs in the Fly Central Complex
}

\author{
- Nicholas D. Kathman and Jessica L. Fox \\ Department of Biology, Case Western Reserve University, Cleveland, Ohio 44106
}

The reduced hindwings of flies, known as halteres, are specialized mechanosensory organs that detect body rotations during flight. Primary afferents of the haltere encode its oscillation frequency linearly over a wide bandwidth and with precise phase-dependent spiking. However, it is not currently known whether information from haltere primary afferent neurons is sent to higher brain centers where sensory information about body position could be used in decision making, or whether precise spike timing is useful beyond the peripheral circuits that drive wing movements. We show that in cells in the central brain, the timing and rates of neural spiking can be modulated by sensory input from experimental haltere movements (driven by a servomotor). Using multichannel extracellular recording in restrained flesh flies (Sarcophaga bullata of both sexes), we examined responses of central complex cells to a range of haltere oscillation frequencies alone, and in combination with visual motion speeds and directions. Haltere-responsive units fell into multiple response classes, including those responding to any haltere motion and others with firing rates linearly related to the haltere frequency. Cells with multisensory responses showed higher firing rates than the sum of the unisensory responses at higher haltere frequencies. They also maintained visual properties, such as directional selectivity, while increasing response gain nonlinearly with haltere frequency. Although haltere inputs have been described extensively in the context of rapid locomotion control, we find haltere sensory information in a brain region known to be involved in slower, higher-order behaviors, such as navigation.

Key words: central complex; fly; haltere; mechanoreception; multimodal integration

Significance Statement

Many animals use vision for navigation; however, these cues must be interpreted in the context of the body's position. In mammalian brains, hippocampal cells combine visual and vestibular information to encode head direction. A region of the arthropod brain, known as the central complex (CX), similarly encodes heading information, but it is unknown whether proprioceptive information is integrated here as well. We show that CX neurons respond to input from halteres, specialized proprioceptors in flies that detect body rotations. These neurons also respond to visual input, providing one of the few examples of multiple sensory modalities represented in individual CX cells. Haltere stimulation modifies neural responses to visual signals, providing a mechanism for integrating vision with proprioception.

\section{Introduction}

Moving animals require information about the movement of their bodies in space. Many animals use vision to guide behavior, but these visual signals often must be interpreted in the context of

\footnotetext{
Received July 12, 2018; revised Jan. 28, 2019; accepted Jan. 30, 2019.

Author contributions: N.D.K. and J.L.F. designed research; N.D.K. performed research; N.D.K. and J.L.F. analyzed data; N.D.K. wrote the first draft of the paper; N.D.K. and J.L.F. edited the paper; N.D.K. and J.L.F. wrote the paper.

This work was supported by Air Force Office of Scientific Research Grants (FA9550-14-0398 and FA9550-16-10165) to J.L.F. We thank Rebekka Bamert for fly artwork used in the figures, and Roy Ritzmann, Alexandra Yarger, and Michael Rauscher for helpful discussion.

The authors declare no competing financial interests.

Correspondence should be addressed to Jessica L. Fox at jlf88@case.edu.

N. D. Kathman's present address: NYU Neuroscience Institute, New York University Langone Medical Center, New York, NY 10016.

https://doi.org/10.1523/JNEUROSCl.1707-18.2019

Copyright $\odot 2019$ the authors
}

body position and trajectory. In mammals, the vestibular system provides acceleration information used in controlling movements. These movements include fast oculomotor eye positioning to stabilize gaze as the head or body moves in space (Lanman et al., 1978), as well as higher-order behaviors such as spatial navigation (Chen et al., 2013; Jacob et al., 2014). Though input from proprioceptors is essential to many vertebrate behaviors, there is little known about how invertebrates integrate bodily senses with external information for decision-making and control.

In flies, stable flight requires proprioceptive organs called halteres that detect body rotations (Yarger and Fox, 2016). Rapid flight maneuvers used in visually guided behaviors (Land and Collett, 1974) require fast feedback about body position, with delays no longer than tens of milliseconds (Dickinson and Muijres, 2016). Haltere mechanoreceptor neurons synapse directly 
onto wing-steering motoneurons (Fayyazuddin and Dickinson, 1996) to facilitate this control. Haltere afferents also synapse onto neck motoneurons that rotate the head (Strausfeld and Seyan, 1985; Huston and Krapp, 2009) to stabilize gaze (Hengstenberg, 1993). Haltere inputs also synapse in many locations in the thoracic and subesophageal ganglia (Chan and Dickinson, 1996), but the subsequent connections of interneurons that may receive this input are not known.

It is possible that haltere information remains peripheral only, synapsing on motoneurons and bypassing interneurons or central integration entirely. Our current understanding of the halteres' influence on behavior suggests that direct connections between haltere primary afferents and wing or neck motoneurons would be sufficient to drive the wing and head reflexes observed in flight. However, the possibility remains that haltere input is integrated or stored by neurons in the CNS. Both visual (Srinivasan, 2000; Homberg et al., 2011; Green et al., 2017; Turner-Evans et al., 2017) and proprioceptive (Wehner et al., 1996) inputs to the CNS provide information about how the animal moves in space. Haltere inputs could provide inertial information used for orientation estimations, useful for path integration or other behaviors mediated by the brain (Srinivasan, 2015; Stone et al., 2017).

A candidate brain region that may use body movement information for higher-order behaviors is the central complex (CX). This is a region of the arthropod brain that receives input from multiple sensory modalities, including visual (in locusts: Heinze and Homberg, 2007; cockroaches: Kathman et al., 2014; bees: Stone et al., 2017; dung beetles: el Jundi et al., 2015; monarch butterflies: Heinze and Reppert, 2011; fruit flies: Seelig and Jayaraman, 2013; and flesh flies: Phillips-Portillo, 2012) and tactile information (Ritzmann et al., 2008). The CX is known to integrate sensory information (el Jundi et al., 2014; Pegel et al., 2018) and control locomotor output (Strauss and Berg, 2010; Martin et al., 2015). Additionally, cells of the insect CX have been shown to encode heading during walking (Seelig and Jayaraman, 2015; Varga and Ritzmann, 2016; Green et al., 2017). Heading representation persists when no visual cues are available, indicating that some nonvisual input must be present either in or upstream from the CX to provide orientation information. Ascending input from the thorax projects to the lateral accessory lobes (LALs; Namiki and Kanzaki, 2016), which provide inputs into the CX. Together, anatomical, behavioral, and neural evidence suggest that the CX is a strong candidate for the integration of haltere and visual information.

Here, we recorded activity in the flesh fly CX during experimental stimulation of both the visual system and the halteres. We find that firing rates of some CX neurons are modulated by haltere input, and that haltere input can alter responses to visual stimuli. Because haltere input is available in the central brain, flies may be able to use body rotation information for a wider array of behaviors than previously appreciated.

\section{Materials and Methods}

Animal preparation. Adult male and female flesh flies, Sarcophaga bullata (Carolina Biological), were raised in a laboratory colony and used in all experiments. Animals were given food and water ad libitum, and kept on a $12 \mathrm{~h}$ light/dark cycle at $27^{\circ} \mathrm{C}$. Recordings were taken $\sim 6 \mathrm{~h}$ into the light cycle. Animals were anesthetized with ice before wings, legs, and calypters were removed. The fly was placed into a plastic micropipette tip (Fig. 1A) with the top cut to be large enough for only the head to protrude. A ring of wax was placed beneath the head to both fix the head and occlude the animal's vision below the neck. Through a hole in the side of the pipette tip, a small iron filing (100-300 $\mu \mathrm{m}$ diameter) was glued to the exposed haltere with UV curing glue (Loctite 3972, Henckel). A small portion of the cuticle between the compound eyes and rostral to the ocelli was then removed, along with connective tissue, fatty tissue, and the ptilinum, to expose the ventral surface of the brain. Care was taken to avoid damage to the ocellar nerve or optic tracts. Saline (Tryba and Ritzmann, 2000) was added to the head cavity to cover the brain tissue. A copper reference electrode was inserted into a hole made on the side of the head and rested near or dorsal to the brain. Before each experiment, we verified that the fly was able to spontaneously move the haltere close to a natural oscillation frequency $(\sim 150 \mathrm{~Hz})$ with the filing attached.

Electrophysiology. Extracellular recordings were performed using 16channel silicon probes (NeuroNexus A-series $2 \times 2$ tetrodes). The two shanks of each probe were dipped 5-10 times in NeuroTrace CM-DiI Paste DiI paste (Invitrogen) for fluorescent labeling of the probe tracks. Each shank was $15 \mu \mathrm{m}$ thick and $150 \mu \mathrm{m}$ apart from center to center and contains two diamond-shaped iridium recording site tetrodes, also spaced $150 \mu \mathrm{m}$ apart vertically from center to center and $80 \mu \mathrm{m}$ from the tip of the shank. The impedance of each channel was 2-3.5 M 2 . Signals were amplified and recorded (RHD2132 16-channel digital amplifier and RHD2000 interface board, Intan Technologies) using freely-available software (Open Ephys, http://www.open-ephys.org). Unit activity was sampled at $30 \mathrm{kHz}$ and bandpass filtered between 600 and $6000 \mathrm{~Hz}$. Only recording waveforms exceeding a predetermined voltage threshold, individually set for each electrode slightly above the noise envelope, for any channel of the tetrode were saved, and recordings were only taken in probe locations where at least one channel showed spiking rates that appeared to change with haltere stimulation.

Haltere and visual stimulation. During recordings, the iron filing attached to the haltere was oscillated via magnetic coupling to a neodymium magnet glued to the lever arm of a high-frequency servomotor (Aurora Scientific). The haltere was oscillated at a constant frequency for $3 \mathrm{~s}$ (Fig. 1C) over a range of 1-150 Hz. Each frequency was presented 2-4 times. We also linearly increased and decreased the haltere frequency over the same range. The magnet-driven haltere oscillation was an approximation of a natural self-driven haltere oscillation. The stimulus was applied in an orthogonal axis to the longitudinal body axis with similar stroke amplitudes and frequencies (at the highest-frequency trials). Changes in stroke plane were held relatively consistent between animals, but, because of the theorized high sensitivity of the haltere's stroke plane (Nalbach, 1993; Thompson et al., 2009), may likely be eliciting varying populations of campaniform sensilla. More detailed and graphical comparisons can be found in a recent study by Yarger and Fox (2018), who used the same haltere stimulation techniques.

To provide visual stimulation, a hemi-cylindrical array of green LEDs (Mettrix Technology) was placed $7 \mathrm{~cm}$ above the fly's head (Fig. $1 A, B$ ) such that each pixel subtended $\sim 3.5^{\circ}$ of the visual space (Reiser and Dickinson, 2008). The LEDs displayed uniform random distributions of bright and dark pixels (Fig. 1B). The optic flow that would result from thrust and yaw movements was simulated by coherent, perspectivecorrected changes in the locations of the bright dots (Theobald et al., 2010). The visual motion periods ran for a duration of $6 s$ at constant speeds, randomly chosen from a set of three speeds $\left(70,350,700^{\circ} / \mathrm{s}\right)$ in either direction (Fig. 1C). These speeds are in the visually responsive range of blowflies (Hengstenberg, 1993) and fruit flies (Duistermars et al., 2007). Then, all combinations of these parameters (haltere frequencies and visual motion parameters) were presented simultaneously, also for $6 \mathrm{~s}$ each. All parameter combinations were randomized and repeated two to four times. Finally, the magnet was removed from the servomotor and the oscillation stimuli were repeated, to control for possible offtarget visual or mechanical effects of the motor's movements. Control signals for both stimuli were recorded by the data acquisition board along with the neural signal.

Experimental design and statistical analysis. Voltage data from each tetrode were sorted offline into unit clusters using Offline Sorter (Plexon). Automated $k$-means clustering was first used to roughly separate unit clusters, followed by manual cluster editing using various waveform features (e.g., the first three principal components and peak-valley ratios of all four electrodes over time). Although extracellular unit separation cannot provide certainty of single-cell isolation, cluster selection 


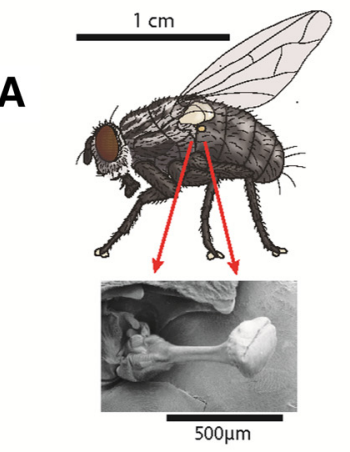

C

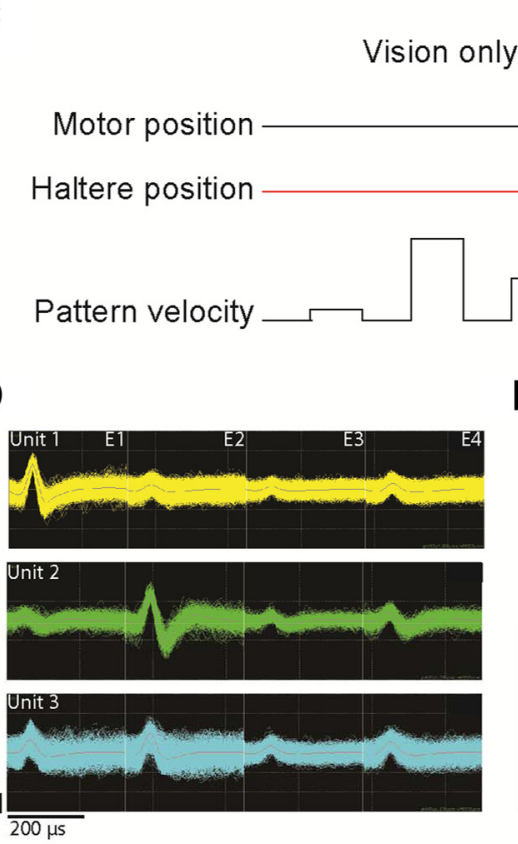

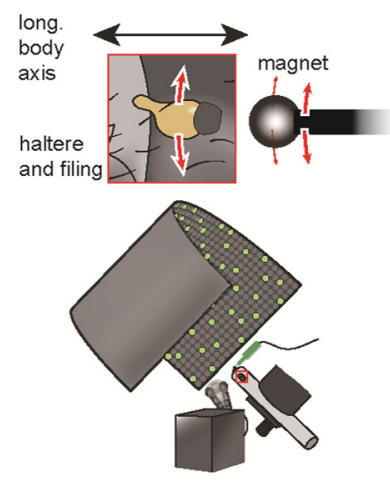

B
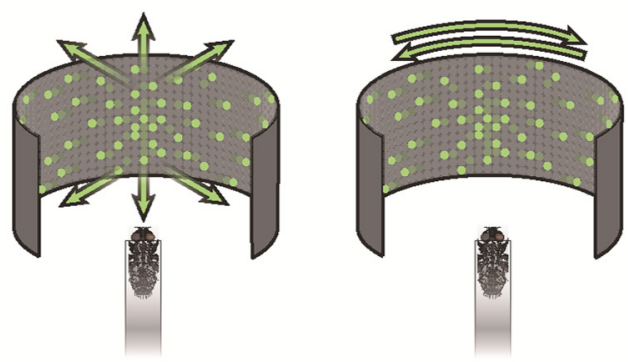

Haltere + visual motion
Magnet removed control

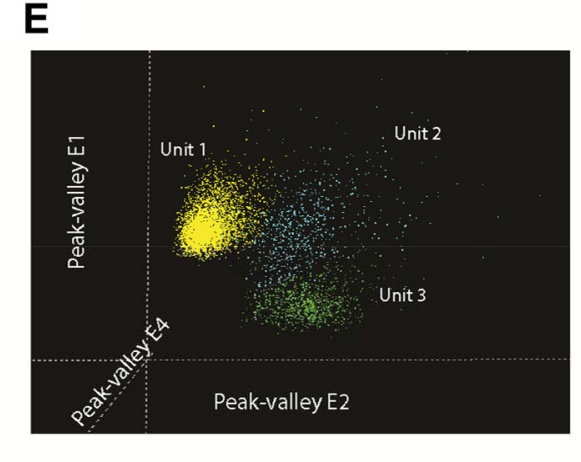

$\mathbf{F}$

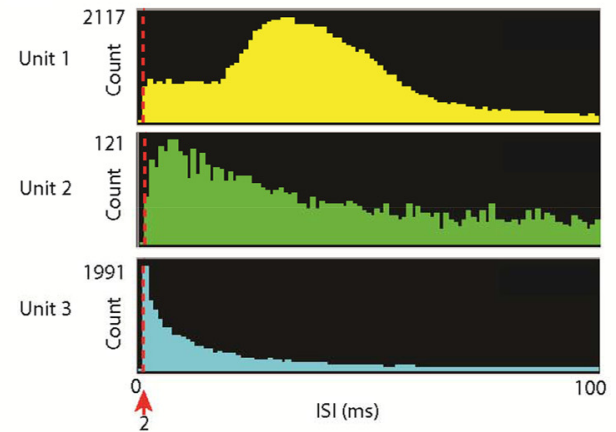

Figure 1. Experimental setup. $A$, Depiction of a flesh fly with scanning electron micrograph of a haltere, next to a schematic of the recording setup (right). A servomotor drives a magnet that oscillates the haltere above and below a central resting position and in a plane perpendicular to the longitudinal body axis via an iron filing glued on the bulb (inset). An LED arena is placed in front of the fly and spiking activity is recorded from the brain. $\boldsymbol{B}$, A star-field pattern was displayed on the LED arena and animated to simulate translational motion (right) and rotational motion in the yaw axis (left). C, Haltere and visual stimuli were presented, varying oscillation frequency and visual speed both independently and simultaneously. Multiple trials of each combination of parameters were presented randomly. After all experimental trials, the magnet was removed from the motor and the stimuli were presented again as a control. $\boldsymbol{D}$, Overlaid waveforms of all sampled spikes in a recording from all four electrodes (e1- e4) in one tetrode, sorted by waveform properties into three units (yellow, green, and blue). Each column has waveforms recorded from each electrode of the tetrode. $E$, Unit separation shown by plotting the peak-valley ratio from electrodes 2,1 , and 4 on the $x-, y$-, and $z$-axes, respectively. Each point represents one spike. Spikes from the recording that were not clustered into these three units are not shown. $F$, ISI histograms of the three sorted units. Each unit has $<2 \%$ of ISIs $<2 \mathrm{~ms}$ (based on refractory period; red dashed line and red arrow). Unit $1=0.2 \%$, unit $2=0.1 \%$, unit $3=1.7 \%$.

was very conservative (Fig. $1 D-F$ ): we discarded any unit with $>2 \%$ of spikes with interspike intervals (ISIs) $<2 \mathrm{~ms}$, or any unit that did not maintain separation or spike waveform consistency throughout the experiment (often $1-3 \mathrm{~h}$ of recording time).

After unit sorting, spike times were imported into MATLAB (MathWorks), where all further data analysis was performed. Instantaneous firing rates were estimated by convolving spike events with a Gaussian kernel function $(\sigma=150 \mathrm{~ms})$. Mean firing rates were found for all trials of each stimulus type (e.g., 1 haltere frequency) by aligning the sample times to stimulus events.

Because we observed strong transient responses consistently near the beginning of visual or haltere stimulus onsets in most units (see Fig. $3 A, B)$, response duration was described as the peak width at half-height of the largest local maxima of mean firing rate during the stimulus period for each stimulus frequency. The first term of the response window was set to zero if the peak width from the smoothed data began before stimulus onset. A standard time window, determined by the median response window for all frequencies, was used to measure response magnitude across different stimulus variables. Only response windows of frequencies where the activity during this time window was significantly different from baseline (Wilcoxon signed rank test for paired samples, $\alpha=0.05$ ) were used in calculating the standardized response window. This was found to reliably predict a window of time that encompassed the largest firing rate changes across all stimulus frequencies (refer to heat maps in Fig. 4).

A unit was classified as responsive if the firing rate (number of spikes/ time for each trial) during this standardized window $\left(R_{\mathrm{t}}\right)$ were significantly different from the firing rates immediately before the stimulus onset $\left(R_{0}\right)$ for all trials of a given stimulus parameter (Wilcoxon signed rank test for paired samples, $\alpha=0.05$ ). When comparing responses across stimulus parameters or across units, our metric of response was $\left(R_{\mathrm{t}}-R_{0}\right) / \bar{R}_{0}$, or $\Delta R / R$ in Figure $4 . \bar{R}_{0}$ is the mean baseline firing rate for the unit for all trials and all stimulus conditions. Visual responses were found using the same response window calculation as haltere responses. 
A

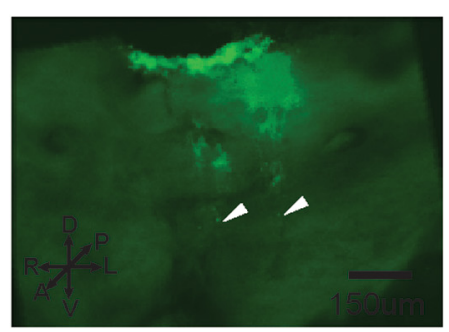

B
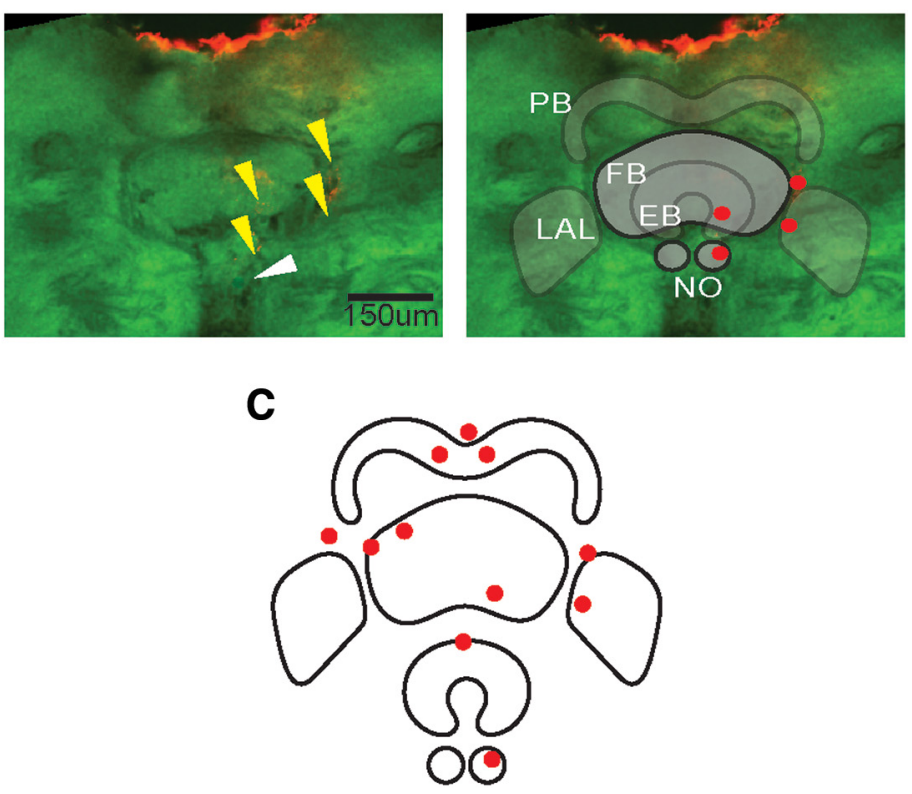

Figure 2. Probe tracks were determined with 3D confocal imaging. $\boldsymbol{A}$, Full image stacks of $z$-plane images show probe tracks (bright green). Ends of probe tracks are indicated with white arrows (left). Recording site positions were determined in relation to the end of the dye track (white arrows) and the dimensions of the recording probes (white diamonds). $\boldsymbol{B}$, An optical slice (left) of an example brain with red dye deposited where the probes were positioned (yellow arrows) in relation to the $C X$, and a representation of the $\mathrm{CX}$ (right) with red dots to mark tetrode locations in the z-plane of the highlighted substructures. In this example, the $\mathrm{FB}$ and $\mathrm{NO}$ are in the foreground, with four recording site locations indicated at that plane, and the PB, EB, and LAL are in relief. $C$, All recording sites of all tetrodes across all animals at the z-plane of the given substructure.

Additionally, a post-peak response was found for all visual and multimodal responses, which used a response window beginning at the end of the peak response window and ending at the end of stimulus presentation (6s).

$P$ values for significance of correlation of unit response and haltere frequency was found using a one-sample $t$ test for the Pearson's correlation coefficient, $r$. The test statistic is given by the following: $t$ $=r \sqrt{\frac{n-2}{1-r^{2}}}$, where $n=$ number of samples. No statistical comparisons were performed repeatedly for a given hypothesis test; therefore, no multiple-comparisons corrections were used.

Spike timing precision relative to the haltere stimulus. To determine whether a unit was firing at a specific phase relative to the haltere oscillation, we measured the phase of each spike with respect to the sinusoid stimulus, and then found the length of the mean vector of these phases. Statistical significance was determined by a Rayleigh $z$ test (Batschelet, 1981). We considered a unit to be phase-locked if it showed significant $(\alpha=0.05)$ phase-locking only during the stimulus period, and not during the equivalent time interval immediately before the stimulus began or during the control experiment with the magnet removed.

Histology. Histology followed the methods by Kathman et al., 2014. Briefly, after recordings concluded, we removed the brain and then fixed, dehydrated, cleared the tissue. Brains were then imaged using a confocal microscope (TCS SP8 gated STED, Leica). Assessments of three- dimensional recording site locations were made based on the location of dyed tracks left from the probes (Fig. 2). Following recordings, optical sections were taken from each brain. Some dye adheres to the surface of the brain upon probe entry (Fig. $2 A, B$ ), and dye tracks in the interior of the brain indicate the location and direction of the probe's progression. Recording sites were determined by finding the location $80 \mu \mathrm{m}$ above the termination of the dye track, as tetrodes were located at this distance from the end of the probe. Substructures present in the plane of section where dye was the appropriate distance from the tip were considered to be the location of the recording site. This provides a 3D location of the recording site, which can be represented in $2 \mathrm{D}$ by overlaying the CX structures present in that slice (Fig. 2B). Probes were never retracted before recording.

\section{Results}

Extracellular multichannel recordings were taken from five flesh flies. During each recording, a randomized series of stimuli was presented to the animal. Either the haltere was driven by a motor via a magnet and iron filing glued to the haltere (Fig. $1 A$ ) or visual motion from an LED display was presented to the animal (Fig. $1 B$ ), or both stimuli were presented simultaneously (Fig. 1C). These recordings yielded 49 neural units that modulated their responses when the haltere was oscillated, where their instantaneous firing rate was above or below a $99 \%$ confidence interval of the unit's mean firing rate during the stimulus presentation. These units were within or on the margin of the CX with distinct waveform characteristics from four groups of four recording electrodes used for each animal. Unit isolation was based on waveform characteristics and interspike interval distribution (Fig. 1D-F). Across all animals, 26 units were recorded in or near the protocerebral bridge (PB), 5 in the fanshaped body (FB), 2 in the ellipsoid body (EB), 5 in the left noduli (NO), 11 in the left LAL, and 4 in the right LAL (Fig. $2 C)$. Only recordings with units responding to haltere motion were included in our analysis.

To control for possible off-target visual or mechanical effects of the motor's movements, the magnet was removed from the motor and the oscillation stimuli were repeated. In magnetremoved trials, the motor oscillated but the haltere remained still. Four units had significant changes in firing rate in both experimental and control trials, and these units were excluded from analysis. Though there remains the possibility that the observed CX responses described below are because of magnetoreception and not haltere mechanosensation, this seems unlikely: magnetoreceptors in flies have been challenging to characterize (Nordmann et al., 2017), may respond only to low frequencies (3-50 $\mathrm{Hz}$; Fedele et al., 2014), and have been shown to play a role in a limited number of behaviors (Gegear et al., 2008; Fedele et al., 2014). 
A
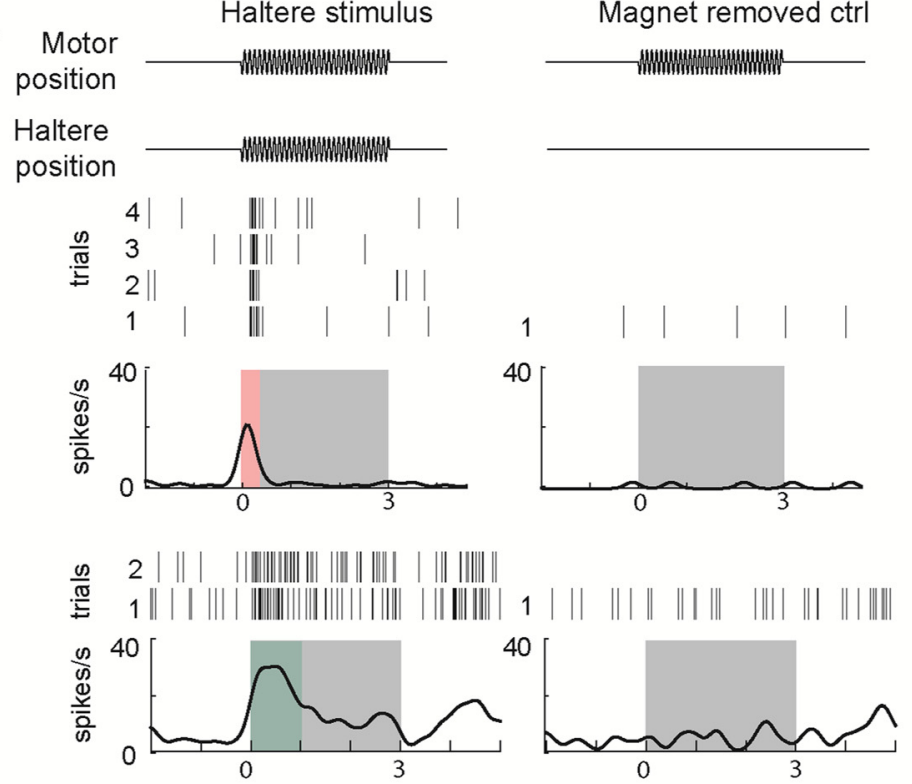

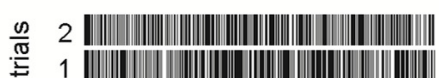

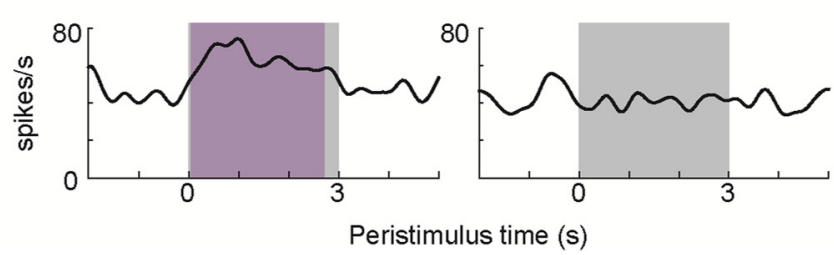

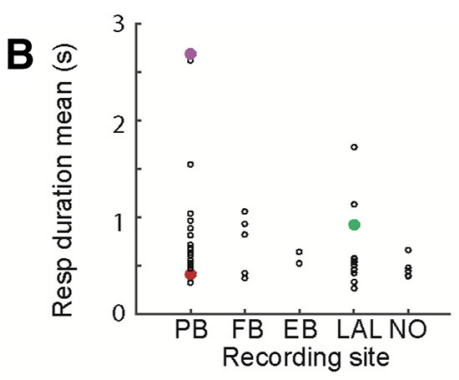
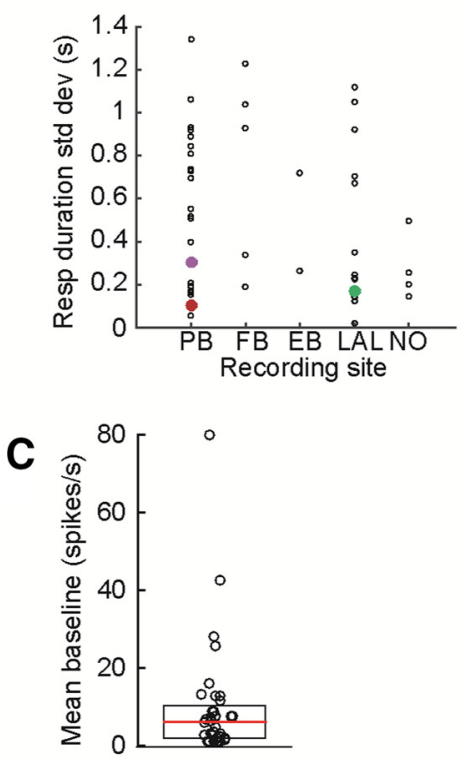

Figure 3. CX cells respond to haltere oscillations with varied duration. $\boldsymbol{A}$, Peristimulus response of three example units to haltere oscillation trials (left column), and control trials in which the magnet is removed from the motor (right column). The motor is oscillating at $60 \mathrm{~Hz}$ in these examples, as represented by a schematic (top). Three units with significant responses (top to bottom: $p=$ $0.0067,0.036,0.040$ ) of different durations are depicted with raster plots showing spike times from the $2-4$ trials of $60 \mathrm{~Hz}$ haltere oscillation and one trial of the control experiment. Mean firing rate (black line) and response duration (colored box) are shown in relation to the stimulus time for all trials (gray box). $\boldsymbol{B}$, Mean (top) and SD (bottom) of response duration across all stimulus frequencies did not correlate with recording site location. Colored points indicate data from the three representative cells on the left. Median response duration across all units was $0.70 \mathrm{~s}$. $\boldsymbol{C}$, Distribution of baseline firing rate for all responding units. Median (red line) is 4.81 spikes/s, first and third quartiles (black box) are 0.71 and 9.71 spikes/s, respectively.

\section{CX cells respond to haltere oscillations with varied duration} Our analysis identified 36 of $49(73 \%)$ recorded units that responded to haltere oscillations with a significant change in firing rate during a time period defined by the median response duration compared with baseline firing rate (Wilcoxon rank sum test for paired samples, $\alpha=0.05$ ). Note that recordings were continued if at least some units responded to haltere oscillations; therefore, this percentage does not necessarily reflect the distribution of haltere-responsive cells in the CX. Thirty-four of the 36 responding units showed increases in firing rate during the stimulus, predominantly as a phasic response at the onset of motion (Fig. $3 A, B$ ) for all stimulus trials and no response during the control trials.

Unlike haltere signals from the primary afferents (Fox and Daniel, 2008), which persist as long as the haltere is oscillating, unit responses were typically brief, beginning at the stimulus onset and lasting $<1 \mathrm{~s}$ (median $=0.70 \mathrm{~s})$. Although most unit responses were phasic, two units responded for the duration of the $3 \mathrm{~s}$ stimulus (Fig. $3 \mathrm{~A}$, bottom unit). Calculated response durations for these units were slightly $<3 \mathrm{~s}$ because of smoothing effects on temporal precision.

Unit response durations were variable when analyzing all stimulus frequencies together (Fig. $3 B$, bottom). We analyzed response windows for each stimulus frequency tested and found no relationship between mean or variance and recording site lo- cation (Fig. 3B). The varying response duration may have implications for a transformation from the timing code in the periphery (Fox et al., 2010) to a rate code in the central brain. This also may affect modulation of other sensory responses moderated by the CX, as described later.

Units had a median baseline firing rate of 4.81 spikes/s (Fig. $3 C)$. Of the nine units with baseline firing rates in the top quartile (Fig. 3C, units plotted above the box), two units were those with long response durations, and two units significantly decreased their firing rate transiently at the onset of haltere motion for some haltere frequencies (Wilcoxon rank sum test for paired samples, $\alpha=0.05)$. There was no correlation between baseline firing rate and recording site location.

\section{Some CX units signal haltere activation and others encode haltere frequency}

To quantify the magnitude of the transient firing rate changes in the responding units, a standardized response time window was determined for each unit to be used for all trials (Fig. 4A). This allowed the comparison of firing rates between units with different durations of responses, as seen in Figure $3 A$. We used the median response periods for all stimulus frequencies as the standardized time window to measure responses across frequencies, which were then normalized by baseline firing rate $(\Delta R / R)$. 

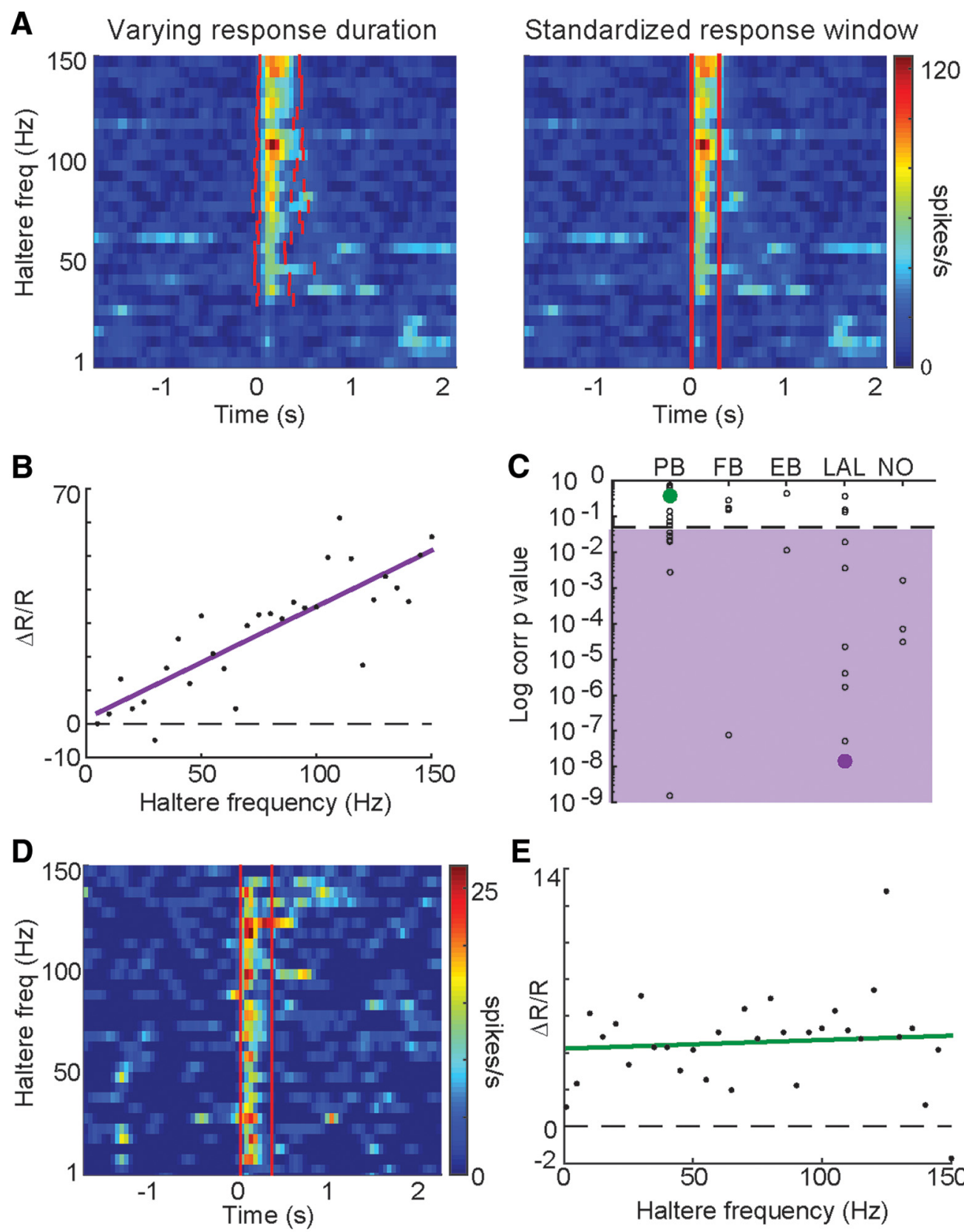

E
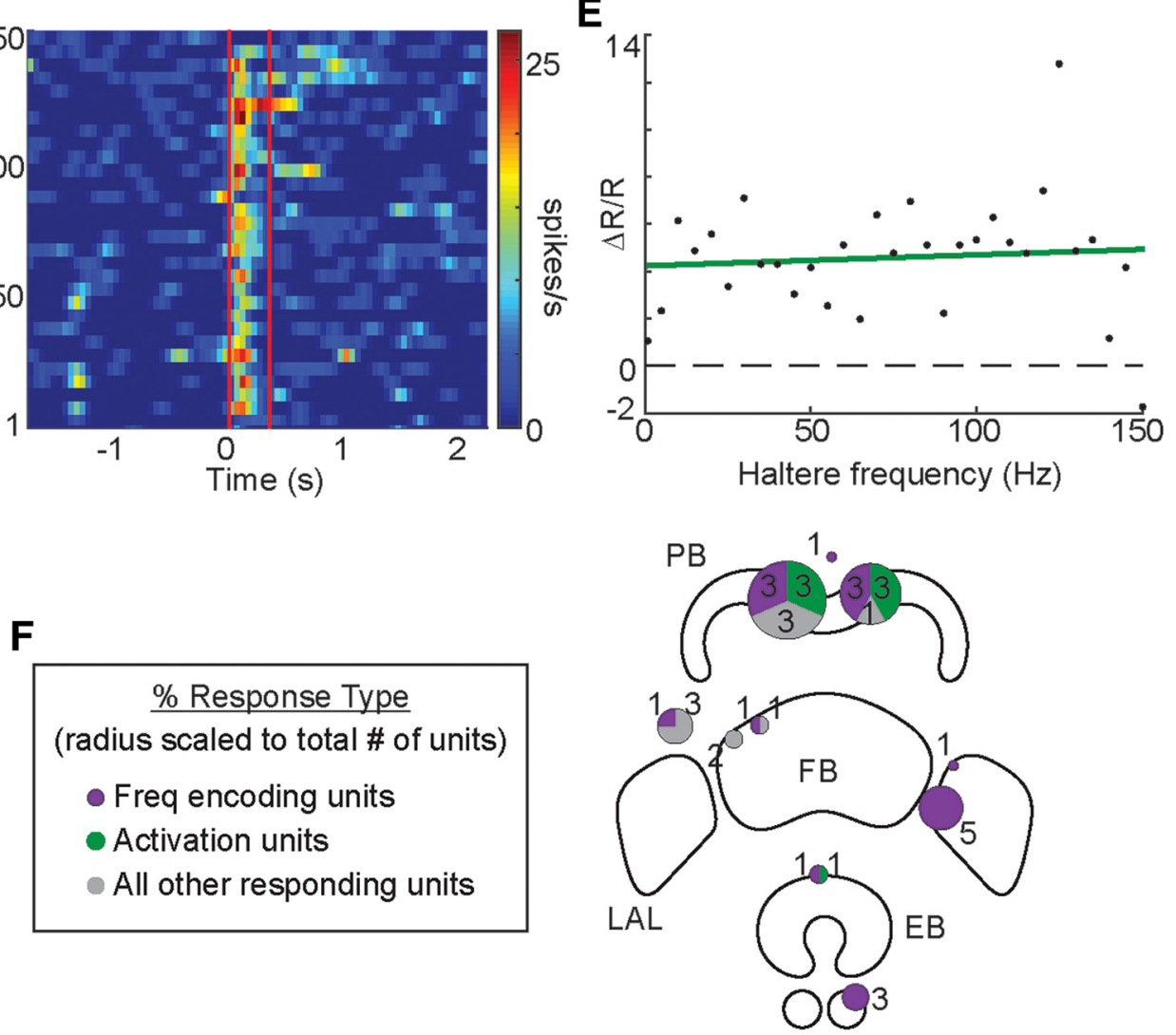

NO

Figure 4. Some $C X$ units signal haltere activation and others encode haltere frequency. $A$, Unit responses with varying response durations (left) were quantified with a standardized response window (right) based on the median of the mean response durations for all haltere frequencies. $\boldsymbol{B}$, The normalized response magnitudes, $\Delta R / R$, for this frequency encoding unit are linearly correlated with haltere frequency $(p=1.19 \mathrm{e}-08)$. A best-fit line, by least squares, is shown in purple. $C$, Distribution of significance of correlation ( $p$ value on a log scale) for all haltere-responding units, grouped by the substructure the recording site was in or near. Unit $p$ values in the purple region are significantly correlated to haltere frequency $(p<0.05)$. P values (Figure legend continues.) 
Nineteen units showed a linear relationship between haltere frequency and firing rate (Fig. $4 A, B$ ). In these units, the normalized mean response was significantly correlated with the stimulus frequency (one-sample $t$ test, $p<0.05$; Fig. $4 C$, purple shaded area); 18 of the 19 units showed a positive correlation. The one unit with a negative correlation showed inhibitory responses; thus, the decrease in firing rate associated with the inhibitory response was positively correlated with haltere frequency. We refer to these units as "frequency encoding units".

To best classify the response relationship with haltere frequency, we also fit quadratic, cubic, and sigmoid models to the data. We used an Akaike information criterion (AICc; with a correction for limited sample size) to compare relative fit for each model (Burnham and Anderson, 1998). Using the conservative threshold for difference in AICc values of four (Burnham and Anderson, 2004), four units with significant frequency correlations lost significantly less information when using a quadratic model (i.e., the quadratic model was a better fit than a linear model; with the difference in AICc values between 4.00 and 20.22). One unit was better fit by a sigmoid model. Twenty-six units showed a best fit to a linear model, which include the 19 units that showed correlation. Therefore, we limited further analysis to linear models and continued to use the more conservative correlation criteria to categorize a frequency relationship.

The remaining 17 units that responded to the stimuli had no significant correlation with frequency (Fig. $4 C$, units above the dashed line; $p>0.05)$. Of these units, seven units still responded to $>75 \%$ of the frequencies tested (Fig. $4 D, E$ ). We refer to these units as "activation units" as they respond to the haltere's activity but are agnostic to its frequency. One of these units was inhibited by haltere input, with reduced firing rate in response to the stimulus.

Of the 19 frequency encoding units, nine were found in the $\mathrm{LAL}$, four in the $\mathrm{NO}$, four in the $\mathrm{PB}$, one in the $\mathrm{EB}$, and one in the FB (Fig. $4 F$ ). Of the responding units in these regions, they represent $90 \%$ of the units recorded in the LAL and $100 \%$ of those in the NO. In comparison, $24 \%$ of PB units showed this trend, as did $50 \%$ of $\mathrm{EB}$ and $\mathrm{FB}$ units, with only two units found in each. Activation units were almost exclusively found in the PB (6 of 7), with only one unit found on the margin of the EB.

The highest frequency trials in these experiments $(150 \mathrm{~Hz})$ were an approximation of self-driven haltere frequency during tethered flight ( $168 \mathrm{~Hz}$; Hall et al., 2015) and in free walking (173 $\mathrm{Hz}$ with variable frequencies; Yarger and Fox, 2018). Because haltere oscillations at the beginning and end of each oscillation bout in both flight and walking are slower and more variable than their peak oscillation frequency, it is possible that frequency encoding neurons of the CX are sensitive to changes in the haltere's oscillation patterns upon changes in behavioral state.

\section{Haltere stimulation entrains spike timing in some CX units} When the haltere is oscillated, the primary afferent neurons of the haltere's campaniform sensilla fire spikes that are precisely timed

\footnotetext{
(Figure legend continued.) for units shown in $\boldsymbol{B}$ and $\boldsymbol{D}$ are shown by filled purple and green circles, respectively. $\boldsymbol{D}$, Example of an activation unit. $\boldsymbol{E}$, Responses in the unit shown in $\boldsymbol{D}$ were not correlated ( $p=0.65$, green best-fit line) with haltere frequency but were significantly higher than baseline (paired $t$ test, $p=3.1 \times 10^{-10}$ ) for $>75 \%$ of the frequencies tested. $\boldsymbol{F}$, Schematic of the unit classification locations with proportion of frequency encoding units indicated by purple and activation units indicated by green in a pie chart at recording site. The radius of each pie chart is scaled to the number of responding units found in that recording site (number of units labeled in or next to charts).
}

to the oscillating stimulus (Pringle, 1948; Fox and Daniel, 2008). This precise spike timing is necessary for behaviors organized by peripheral circuits: wing-steering activity is driven by the relative timing of inputs from the haltere and wing mechanosensory neurons (Heide, 1983; Fayyazuddin and Dickinson, 1999). However, such spike timing may not be necessary for behaviors initiated in the brain, which might take place on longer timescales.

We measured the relative phase of each spike to the haltere stimulus to determine whether CX neurons fire at specific times in the haltere oscillation cycle. We found 8 (of 49 total) units that fired at specific phases of the sinusoidal haltere stimulus at one or more stimulation frequencies [Fig. 5A,C; Rayleigh $z$ test on the length of the mean vector (Batschelet, 1981); $\alpha=0.05]$. The activity of these units was not phase-locked during interstimulus periods when the haltere was stationary, nor during the control experiment in which the magnet was removed from the motor. Some units (5 of 8) showed phase-locking activity in trials that were conducted several minutes apart, showing that phaselocking activity is consistent over time (Fig. $5 B$ ).

Units were phase-locked at various frequencies over the range tested. The best-fit line relating the haltere oscillation frequency to the mean spike phase of each unit is suggestive of a direct relationship ( $r=0.35$ ) but is not statistically significant (onesample $t$ test, $p=0.06$ ), and the large variances (and resulting low vector strengths; Fig. $5 C$ ) around the mean phases indicate that the phase-locking activity of the haltere afferent neuron is not faithfully transmitted to the CX.

Though the phase-locking of these eight units was significantly different from random (Batschelet, 1981), the vector strengths were generally low, with only one unit approaching mean vector lengths similar to those measured in primary afferent neurons (Fig. 5C). This unit was found in the lower left LAL. Median vector strengths for six of the eight units was $<0.8$, indicating weak but significant phase-locking. There was also no significant relationship between unit mean phases and stimulus frequency (Fig. 5D). Thus, though information about individual oscillations of the haltere is represented in some cells, the mechanism for encoding haltere information in most units of the CX is likely distinct from the mechanisms used by haltere primary afferent neurons, which rely on precise spike timing to transmit information (Fayyazuddin and Dickinson, 1996; Yarger and Fox, 2018).

\section{Haltere-responsive units also respond to visual motion}

The same units were also tested for responsiveness to wide-field visual motion using a pattern of random dots (Theobald et al., 2010) on an LED arena in front of the restrained fly. The pattern simulated yaw rotation (left or right) or linear thrust translation (backward or forward; Fig. 1B). Animals were presented with randomized trials of unisensory visual motion and multisensory stimuli (simultaneous visual motion and haltere oscillations), with varying speeds of each. Twenty-six of 49 units (67\%) responded to at least one visual stimulus ( $\alpha=0.05$; Fig. $6 A$ ). Of the 36 units that responded to haltere motion, 22 responded to visual motion as well. Four additional units responded only to visual motion. Of the visually responsive units, all responded to linear thrust translation, but only 18 of 26 responded to yaw rotation.

Like the responses to haltere stimuli, responses to visual and multisensory stimuli were both large and phasic at the stimulus onset. However, nine units had tonic increased firing (paired $t$ test of stimulus period after transient compared with baseline, $\alpha=0.05)$ which returned to baseline at the end of the motion stimulus (Fig. 6A). One unit responded tonically for the duration 
A

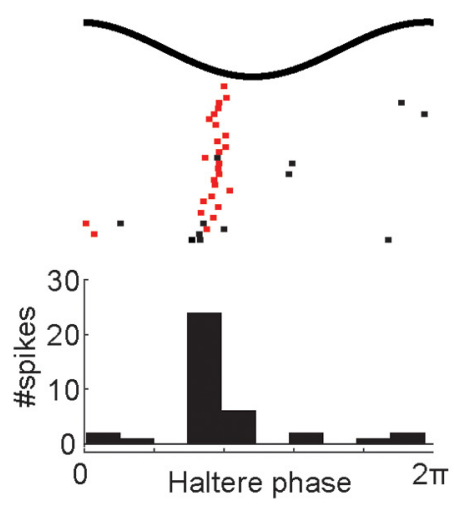

C

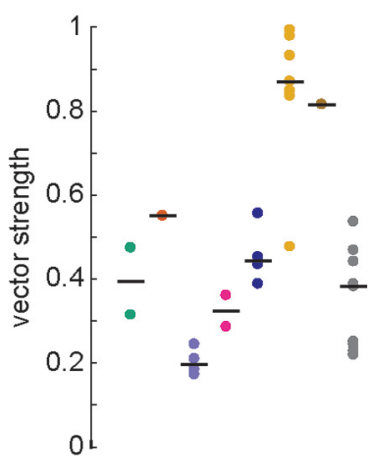

B

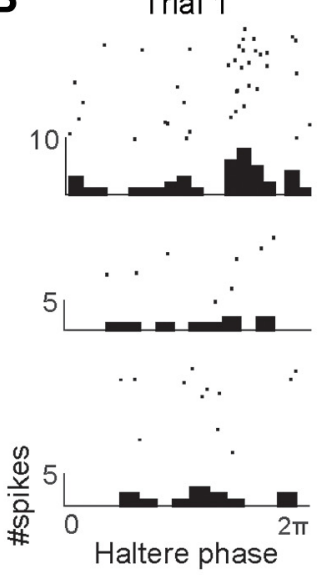

D

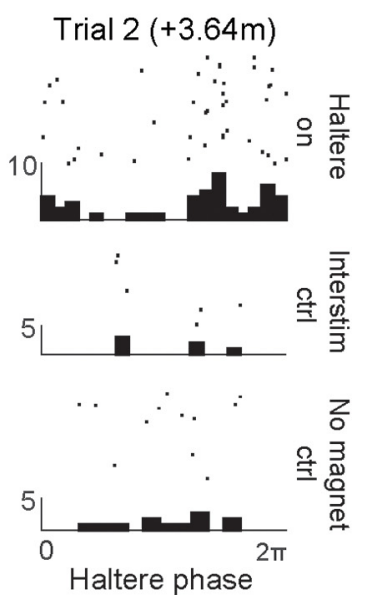

\begin{tabular}{|r|}
\hline Unit \# \\
- 312 \\
- 318 \\
- 321 \\
- 323 \\
- 327 \\
- 421 \\
- 425 \\
- 431 \\
\hline
\end{tabular}

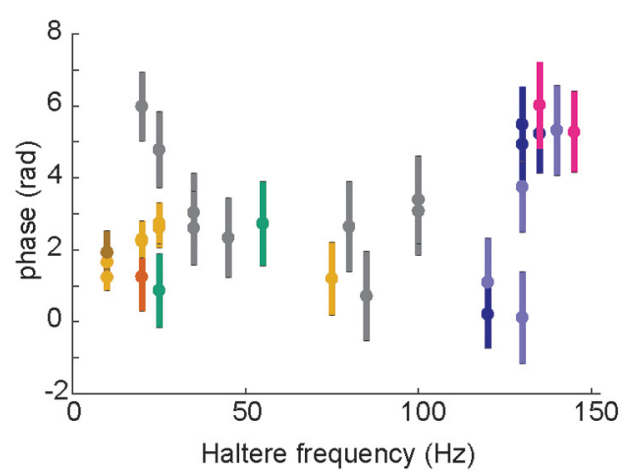

Figure 5. Haltere stimulation entrains spike timing in some $C X$ units. $A$, Raster plot of spike times in response to a $20 \mathrm{~Hz}$ sine wave oscillation of the haltere. The first spike of each stimulation cycle is labeled in red; subsequent spikes are black. $B$, Example of a unit with phase locking in two trials that occurred 3.64 min apart. Left column, first trial; right column: second trial. Top row, Raster plot of responses to 350 cycles of a $130 \mathrm{~Hz}$ sine wave stimulation of the haltere, with histogram of spike phases below. Vector strengths of Trials 1 and 2 were 0.44 and 0.45 , respectively. Middle row, Responses during interstimulus periods in which no haltere stimulation was provided. Bottom row, Responses to 350 cycles of $130 \mathrm{~Hz}$ motion of the servomotor with the magnet removed. Rayleigh $z$ test of vector strength shows significant phase locking $(\alpha=0.05)$ only during haltere stimulation, and not during interstimulus periods or the control experiment with the magnet removed. $C$, Vector strengths of the eight units that showed statistically significant phase-locking activity in at least one trial. $\boldsymbol{D}$, Haltere stimulation frequency and mean phase ( \pm angular deviation).

of the visual motion, and only to forward motion. These longerduration responses are similar to those directionally selective units previously found in the central body of the cockroach CX (Kathman et al., 2014) and noduli tangential neurons of the bee CX (Stone et al., 2017).

Multisensory responses are both sublinearly and superlinearly summed, depending on haltere frequency

When presented with haltere oscillations and visual motion simultaneously, onset response magnitude changed compared with both the visual or haltere responses alone. All of the 22 units with responses to both modalities showed an increase in response magnitude to at least some combination of stimulus parameters (Fig. 6A). Three units showed a decrease in response to multisensory stimuli, and did so only in small subsets of visual speeds and directions.

Although these units often had a larger response to multisensory stimuli, responses were not always linear summations of the unisensory responses. We calculated responses for linear summation analysis by subtracting the mean baseline firing rate from the mean response period firing rate. Six units (Fig. 6B) showed a linear correlation between responses to multisensory stimuli and the sum of the unisensory responses ( $t$ test for correlation, $p=$ $1.0 \times 10^{-5}$ to $4.7 \times 10^{-5}$ ).
The multisensory responses were often synergistic (superlinear) or antagonistic (sublinear), depending on the haltere frequency (Fig. 6C-E). Four of the six units showed a mean sublinear response with $1 \mathrm{~Hz}$ haltere stimuli and superlinear or linear responses at higher frequencies (one-sample $t$ test, $p=$ $7.1 \times 10^{-9}-1.7 \times 10^{-7}$; Fig. $6 E$ ). Four other units showed higher residuals (i.e., the difference of the observed multisensory response from the sum of the unisensory responses) at $100 \mathrm{~Hz}$ than the slower haltere frequencies $\left(p=1.4 \times 10^{-4}\right.$ to $\left.2.7 \times 10^{-9}\right)$, but only two of those units were superlinear at the highfrequency $\left(p=4.9 \times 10^{-5}\right.$ and $\left.2.9 \times 10^{-10}\right)$. Eleven of 22 units had sublinear responses at $1 \mathrm{~Hz}$ haltere frequencies $(p=1.4 \times$ $10^{-14}$ to 0.033 ). No consistent differences in multisensory responses compared with a linear summation, were seen when considering visual parameters, such as speed or direction. This is the case in all units, including those sensitive to both. Preferred direction of units with directional selectivity (discussed in the following section) also did not affect the summation of the responses.

\section{Haltere input modulates responses to visual stimuli}

Some units showed directional selectivity to visual motion, as seen in the CX in previous studies (Phillips-Portillo, 2012; Seelig and Jayaraman, 2013; Kathman et al., 2014), where responses to motion in one direction were significantly different from re- 
A
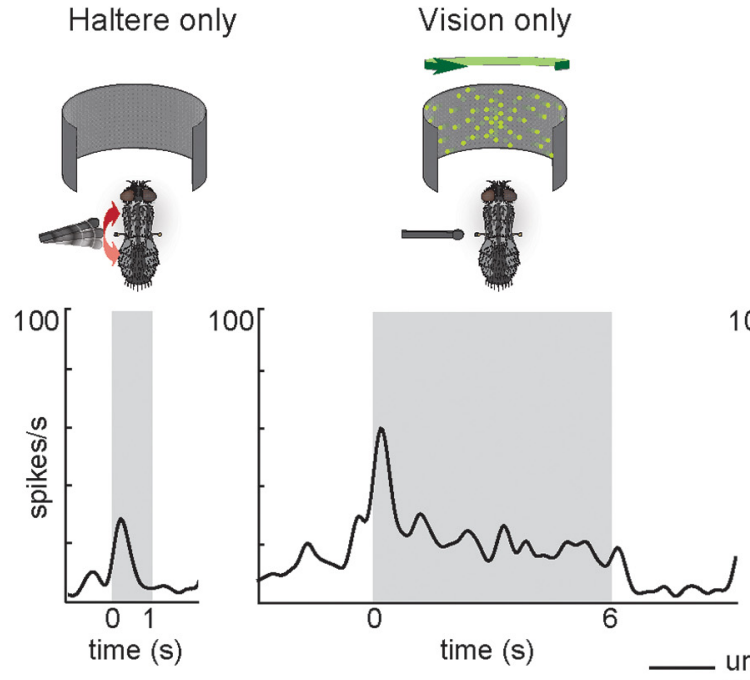

Haltere

C

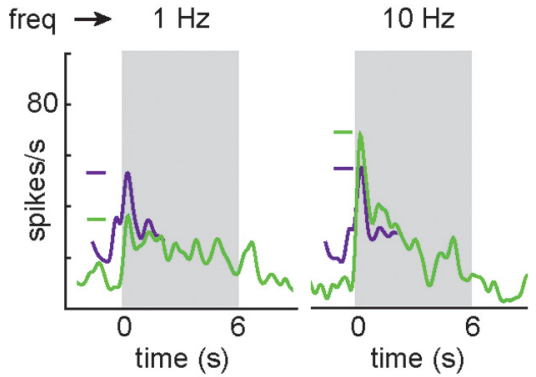

Vision only

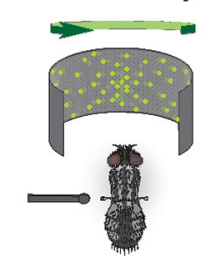

(s) multise

- linear
Haltere + vision

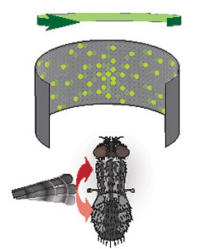

B

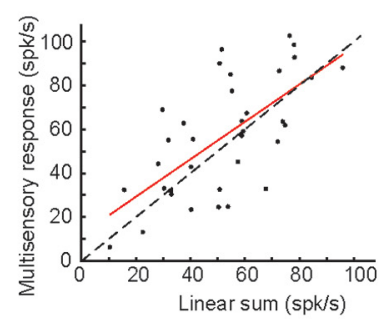

E

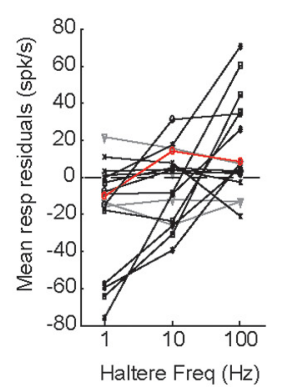

Figure 6. Multisensory responses are both sublinearly and superlinearly summed, depending on haltere frequency. $A, A$ unit response to stimulation of the haltere alone (left), visual motion alone (middle), and both stimuli simultaneously (right, green line). A summation of both unisensory responses is shown in purple ( $100 \mathrm{~Hz}$ haltere 0 scillations and $70^{\circ} / \mathrm{s} \mathrm{left} \mathrm{yaw} \mathrm{visual} \mathrm{rotation).} \mathrm{Gray} \mathrm{box}$ indicates time of stimulus presentation. Dashed box indicates response period used for summation comparisons. All $\boldsymbol{y}$-axes are on the same scale. $\boldsymbol{B}$, Responses from the unit shown in $\boldsymbol{A}$ to all speed, direction, and haltere frequency combinations of multisensory stimuli versus the corresponding linear sum of unisensory stimuli. Baseline firing rate is subtracted from all responses. Dashed diagonal line indicates linear summation. Red line is a least-squares fit linear model $\left(r=0.66\right.$, slope $\left.=0.85, p=1.3 \times 10^{-5}\right)$. C, Sublinear and superlinear summation depends on haltere frequency. Multisensory and linearly summed unisensory responses of the previous unit for three different haltere frequencies (all for $70 \% \mathrm{~s} \mathrm{left} \mathrm{yaw} \mathrm{visual} \mathrm{rotation).} \mathrm{Purple} \mathrm{and} \mathrm{green} \mathrm{dashes} \mathrm{indicate} \mathrm{peak}$ height of corresponding responses. All $y$-axes are on the same scale. $\boldsymbol{D}$, All multisensory response residuals (difference between observed multisensory response and linearly summed unisensory responses) for each visual speed and direction of this unit, separated by haltere frequency. Mean shown in red. Multisensory responses to $1 \mathrm{~Hz}$ haltere oscillations show sublinear summation, $10 \mathrm{~Hz}$ oscillations produce superlinear summation, and $100 \mathrm{~Hz}$ oscillations produce variable results. ${ }^{*} p=0.0053$, two-sample $t$ test. $\boldsymbol{E}$, Mean of residuals across visual speeds at each haltere frequency for all units that respond to both modalities ( $n=20$ units). Units shown in black showed differences between residuals at each frequency (one-way ANOVA, $\alpha=0.05$ ); units shown in gray did not. Unit depicted in $\boldsymbol{D}$ shown in red.

sponses to the opposing direction (Fig. 7A). Eight units showed a significant difference in the response for one direction (with consistent selectivity for at least two of the three speeds tested; twosample $t$ tests, $\alpha=0.05$ ) during either thrust or yaw motion: six only during thrust motion, one only during yaw rotation, and one during both directions of motion. Changing visual speed often influenced directional selectivity, but not in a consistent way between units. An example unit, shown in Figure 7A, has a significantly greater response to forward thrust than backward thrust at all speeds (two-sample $t$ tests, $p=0.041,0.036,0.001$ for high, medium, and low speeds, respectively), and a greater response to forward thrust than to yaw motion in either direction at high speeds ( $p=0.02$ and 0.44 , for left and right directions, respectively). This unit's preferred direction was thus classified as forward thrust.

Units with directional and speed sensitivities maintained these characteristics when the halteres were stimulated, but with increased response magnitudes at higher haltere frequencies (example unit shown in Fig. $7 B$ ). Of the 22 units with responses to both stimuli modalities, 17 units showed strong modulatory changes with frequency in at least half of the visual speeds tested (one-way ANOVA, $p<0.05$; Fig. $7 C$ ) when comparing the initial phasic responses, which are present in both unisensory responses. In six units, the response during the period after this initial phasic change also was modulated by haltere frequency, but to a lesser degree (Fig. 7D). In this post-phasic response period of this unit, responses to thrust motion (the unit's preferred direction) were increased, but responses to yaw motion were not.

These changes in magnitude were not always linearly correlated with haltere frequency (Fig. $7 C, D$ ). Of the nine units with responses to multisensory stimuli that also showed linear responses to haltere frequency in haltere-only trials, three were not linearly correlated ( $p=0.066-0.92)$ in the multisensory trials for any visual speeds or directions. In these units, the phasic response showed a depression in magnitude at $1 \mathrm{~Hz}$ haltere frequency (compared with the vision-only response), but showed a large increase in response at a slightly faster oscillation of $10 \mathrm{~Hz}$, and maintained this increase at the highest frequency tested, $100 \mathrm{~Hz}$.

These units thus inhibit the visual responses when the haltere moves slowly, and then saturate the responses after the haltere's movement has surpassed a low threshold of at least $10 \mathrm{~Hz}$. Six of the nine linear units with multimodal responses showed no sig- 
A
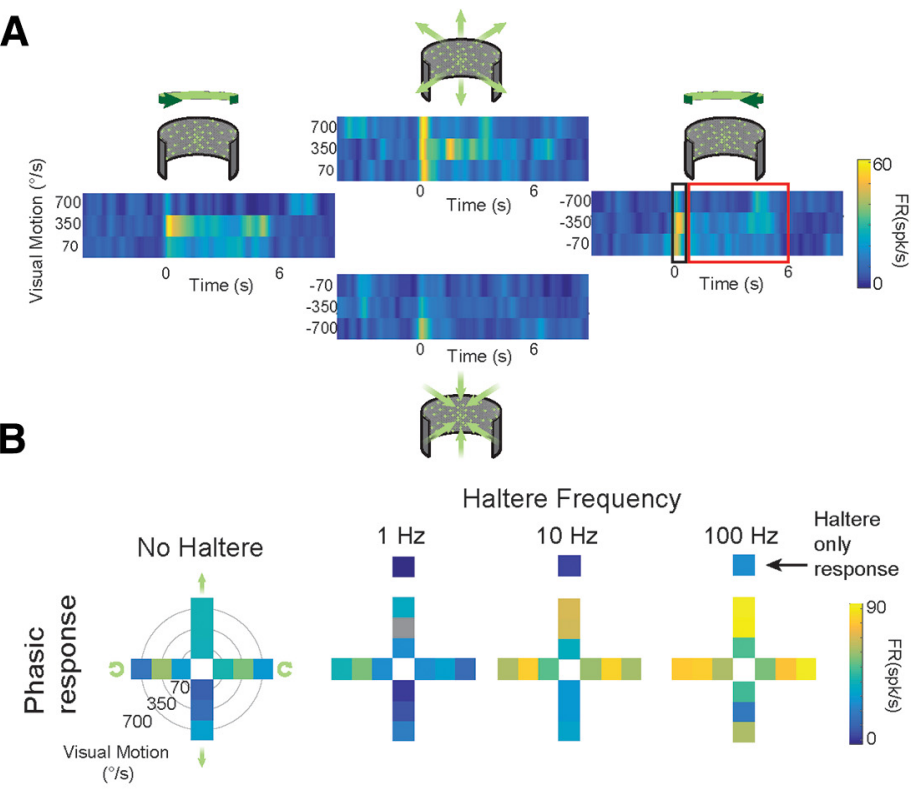

Haltere Frequency
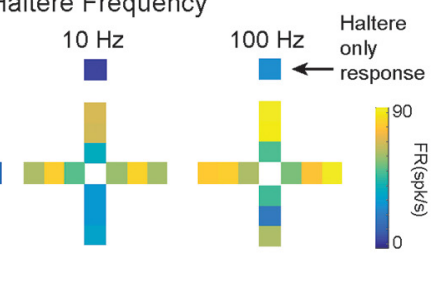

C

Yaw
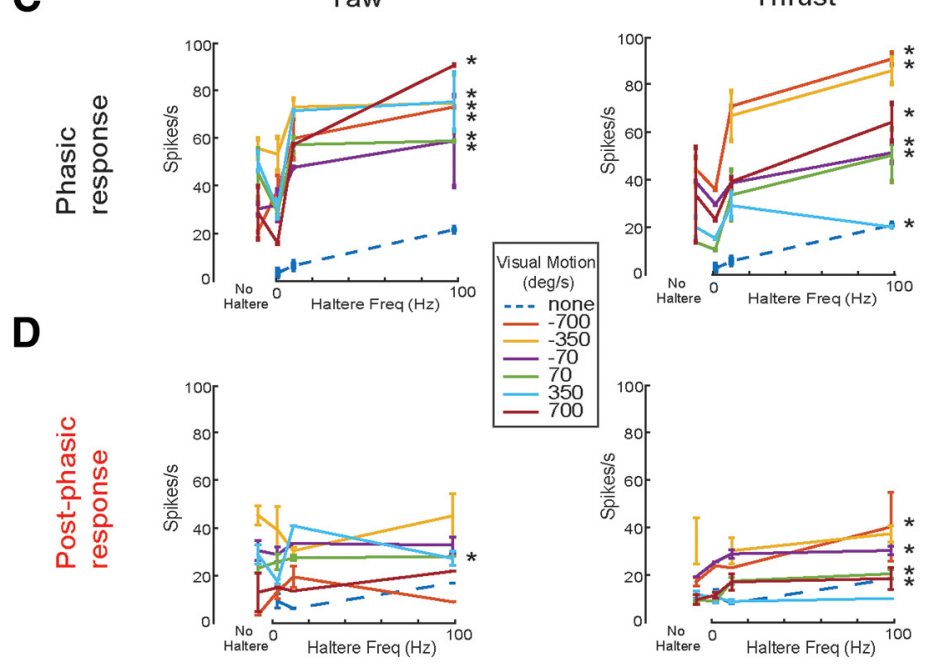

E

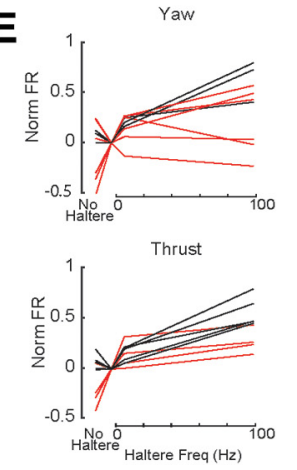

$\mathbf{F}$
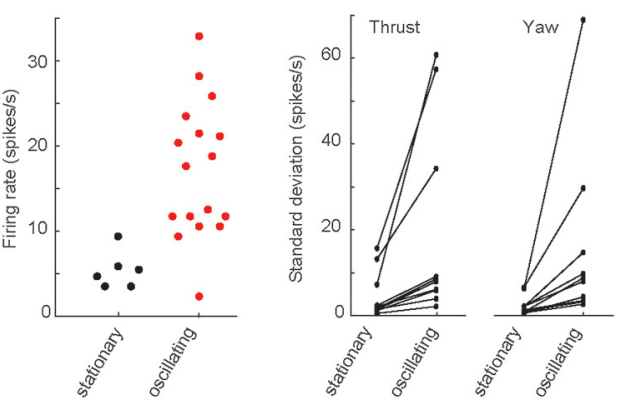

Figure 7. Haltere input modulates responses to visual stimuli. $A$, Directionally selective response of one unit to visual motion in various directions and speeds. There is no haltere stimulation during these trials. Four groups of heat maps show firing rate over time for three speeds at each direction of motion: forward thrust (top), backward thrust (bottom), leftward yaw rotation (left), rightward yaw rotation (right). The motion begins at time 0 and ends at $6 \mathrm{~s}$ for all conditions. Response windows for phasic onset (black) and post-phasic (red) responses indicated by colored boxes. $\boldsymbol{B}$, Visual responses, as changed by haltere frequency. Crossplots using the same coordinate framework as part A for stimulus type. A single square is colored by the response magnitude during the phasic response period at onset (not normalized to baseline). C, Phasic responses versus haltere frequency for all visual speeds and directions (line color), compared with the haltere-only response (dashed line) and visual-only response (leftmost point of each line). All speeds and directions showed significant changes following haltere stimulation ( ${ }^{*}$ one-way ANOVA, $p=0.0008$ 0.0487). D, Post-phasic responses also show modulation of visual response by haltere input, but not in all directions and speeds. Responses to thrust are modulated by haltere input at all speeds but one ( ${ }^{*}$ one-way ANOVA, $p=0.013-0.048$, and 0.45 ). For yaw, nificant difference (paired $t$ test, $p=$ $0.066-0.72$ ) between the 10 and $100 \mathrm{~Hz}$ haltere frequencies across all visual speeds and directions in the yaw direction, and four of these six also showed no difference $(p=0.13-0.79)$ in the thrust direction (Fig. $7 E$ ). Fifteen of the 22 total multimodal units also showed no change between these speeds ( $p=0.071-0.99$ ). In response to visual thrust, there was no significant depression in the post-phasic response, but responses showed a similar nonlinear saturation at $10 \mathrm{~Hz}$.

\section{Haltere movements increase variance of some $\mathrm{CX}$ unit responses to different visual speeds}

We measured firing rates of CX neurons in response to different directions and speeds of visual motion. While we displayed stimuli simulating thrust and yaw movements, we simultaneously oscillated the haltere at 1,10 , or $100 \mathrm{~Hz}$, or left it stationary. Responses to visual motion changed when haltere input was added, as described above, and we noted that responses were generally more variable when the haltere was oscillated (Fig. $7 F$, left). In 10 of 22 units, the variance of responses to different thrust motion speeds was significantly higher when the haltere was oscillated compared with the variance of responses when the haltere was stationary (two-sample $F$ test for equal variances, $\alpha=0.05$, for significant units; Fig. 7F, right). In the other units, response variances did not change with haltere movement (i.e., in none of the units did haltere input decrease variance).

For visual yaw motion, 11 of 22 units had significantly higher variances in responses to different speeds when the haltere was oscillated, and other units did not have significant differences in variance (two-sample $F$ test for equal variances,

\footnotetext{
haltere input changes the response to all but one visual speed $(p=0.0081$ and $0.25-0.98)$. Trial sizes in $\boldsymbol{C}$ and $\boldsymbol{D}$ for each visual speed, direction, and haltere frequency combination varied from 1 to 5 . $E$, Mean response across all visual speeds in the thrust and yaw directions, normalized by the maximum and minimum responses of that unit and aligned by the response at $1 \mathrm{~Hz}$, for all units with linear haltere-only responses. Red response curves show no difference between 100 and 10 $\mathrm{Hz}$ (paired $t$ test, $p>0.05$ ). $\boldsymbol{F}$, Left, Responses to all visual yaw speeds for a single unit when the haltere was stationary or oscillating. Variance in firing rate increases when the haltere is oscillated. Right, SD of responses to different visual motion speeds. Each data point is the SD of responses to seven different visual speeds from a single unit. All units shown have significantly higher SDs when the haltere is oscillating (10 of 22 units for thrust, 11 of 22 units for yaw; $F$ test for equal variances, $\alpha=0.05$ ).
}

$\leftarrow$ 
$p=0.00004-0.046$ for significant units; Fig. $7 F)$. These results suggest that different speeds of visual motion may be more easily discriminated by downstream neurons when the halteres are activated. A higher variance in responses indicates that the unit is expanding its range of firing rates when the haltere is oscillated, making responses to different visual speeds more discriminable by downstream neurons. The increased variance in firing rate may lead to more reliable signaling of visual speed during highfrequency haltere stimulation.

\section{Discussion}

We have shown that information from the gyroscopic halteres of flies is represented in the CX of the brain. The haltere provides essential input that is sent directly to motoneurons without any central processing (Fayyazuddin and Dickinson, 1996), and current understanding of the haltere's role in behavior indicates that the information it provides could remain isolated to the periphery. Our data show that at least some input from the haltere is represented in the central brain. Although we cannot specify the site of integration, integrated information from both the haltere and visual systems are represented in cells of the CX and could be used to guide a broad range of behaviors.

\section{Ascending information from haltere input is represented in CX}

At the base of the haltere lie several groups of campaniform sensilla, each innervated by a single mechanosensory neuron. The major fields of campaniform sensilla all send projections through the cervical connectives into the subesophageal ganglion, where they terminate among the dendrites of the cells that ultimately form the cervical nerve (CN), CN1-CN8 (Strausfeld and Seyan, 1985; Chan and Dickinson, 1996). The cells of the cervical nerve are motoneurons originating in the head that act in concert with neurons of the frontal nerve (originating in the thoracic ganglion), to move the head. Only minor groups of sensors- the small sensilla field $\mathrm{vF} 1$, the single sensillum $\mathrm{dS} 1$, and the sensory hairs on the knob-have projection patterns that fail to ascend through the cervical connectives (Chan and Dickinson, 1996).

For our current understanding of haltere influence on behavior, the small circuits that have been described for head and wing movements are sufficient. Haltere primary afferent axons synapse onto motoneurons of the wing (Fayyazuddin and Dickinson, 1996) and head, either directly or through a single interneuron (Strausfeld and Seyan, 1985). Wing steering and gaze control are known to be directly affected by haltere manipulation or ablation (Hengstenberg, 1988; Dickinson, 1999; Mureli and Fox, 2015; Mureli et al., 2017) and a simple connection from haltere afferents to appropriate motoneurons could explain these behaviors.

We show here that input from haltere afferents can influence firing rates and spike times in neurons of the CX. This information is most likely filtered and processed by interneurons, as there are no direct synaptic connections known between haltere nerve axons and neurons located in the brain. The haltere signal is transformed from the precise, phase-dependent timing code of the haltere primary afferent to a firing rate code. Both the mechanism and substrate of this transformation is unknown, but multisynaptic pathways exist for other inputs into the CX (Pfeiffer et al., 2005; Strausfeld, 2012) and similar transformations are seen in mechanosensory inputs into the brain (Gao and Wehr, 2015). Responses to other thoracic mechanosensory inputs have been found in the central brain. Cells of the LAL of the locust Schistocerca gregaria respond to passive movements of the wing
(Homberg, 1994) and resemble the haltere responses found in this study. Additionally, transient onset responses, similar to the haltere responses shown here, have been observed to antennal deflections in a cockroach CX (Ritzmann et al., 2008). Antennal deflections and haltere oscillations are very different types of sensory information, but the integration of mechanosensation with other information, such as visual inputs, may be a shared function of the CX.

\section{Haltere input has specific effects and is not likely a general arousal or behavioral gate in the $\mathrm{CX}$}

The activity of sensory neurons, even peripheral ones, can be modulated by changes in behavioral state (Chiappe et al., 2010; Maimon et al., 2010; Suver et al., 2012). Could information from the haltere act as an indicator of behavioral state for CX neurons? Certain neck motoneurons in blowflies will fire action potentials only if the halteres are oscillating (Huston and Krapp, 2009), and this "gating" activity suggests that the halteres are used to ensure that head movements are made only during walking or flight (Haag et al., 2010). Similar to neck motoneurons, some units in the CX respond to haltere input but are agnostic to its frequency (Fig. 4D) and could signal that the halteres are oscillating at any speed. We note, however, that in blowflies and flesh flies, the halteres oscillate at similar frequencies during both walking and flying (Hall et al., 2015), and thus, a downstream neuron would likely be unable to determine the specific behavioral state from the haltere frequency. Similarly, the neck motoneurons that show gating activity are not selective for specific haltere frequencies; oscillations as low as $10 \mathrm{~Hz}$ (well below the biological range of haltere oscillation) are sufficient for action potentials. In the peripheral visual system of Drosophila, lobula plate tangential cells are modulated by walking behavior in the absence of haltere movements (Chiappe et al., 2010), indicating that other mechanisms are available to signal behavioral state.

Additionally, fruit flies with ablated halteres are still able to fly on a tether, and the postural changes associated with flight (visually-stimulated head movements and retraction of the front legs) are intact (Mureli et al., 2017). These tethered flies show differences in their responses to specific visual stimuli when their halteres are ablated, suggesting that haltere input does not act as a general flight signal but rather confers specific information. Although the haltere can act as a behavioral gate for a small subset of motoneurons (Huston and Krapp, 2009), it does not appear to be a particularly useful indicator of behavioral state in peripheral neurons.

In the CX, the modulation of activity by the haltere is further indicative of a possible role for the haltere in behavior. We found that changing the frequency of the haltere's movement alone had specific effects on the firing rates of some CX neurons. Though the biological significance of this sensitivity remains to be shown, a coding paradigm could use a frequency relationship to translate information to or from the oscillatory coding scheme of the peripheral flight signals. Wingbeat frequency, which is mechanically coupled to the haltere frequency (Deora et al., 2015), also may be indicative of flight speeds (Frye and Dickinson, 2004). Differences in responses to thrust visual motion compared with yaw rotations, during multisensory stimulation could also relate flight speed information via haltere frequency.

It is possible that haltere frequency may provide behavioral context to visual motion-dependent tasks of the CX. When presented with visual motion stimuli concurrently with haltere motion, the frequency dependency is greatly reduced, nearing saturation at a much lower frequency. Visual responses of the CX 
are known to be modulated by behavioral states, such as flight or quiescence (Weir et al., 2014). The dynamic range of haltere frequency dependence in the units found in this study was greatly reduced and shifted to lower frequencies when visual motion inputs are available. It is possible that for some tasks mediated by the CX, unlike head tracking or saccadic turning, haltere activation above a relatively low threshold enhances the visual motion signal for that context.

\section{Possible functions for haltere-visual integration in the central brain}

Flies use input from the halteres to steer their wings and move their heads, and these behaviors are presently understood to require only small, peripheral circuits. For wing-steering behavior, haltere primary afferent neurons synapse directly onto a motoneuron (Fayyazuddin and Dickinson, 1996), creating a fastacting circuit with a minimum of connections. Thus, there does not appear to be a compelling behavioral requirement for the representation of haltere information in the brain, where the slower, multisynaptic signal would be delayed and less useful for flight control. Though our data do not immediately point to a specific behavior that relies on haltere-related CX activity, there are multiple fly behaviors that might benefit from the integration of haltere input with other information.

The presence of haltere-related activity in neurons of the CX provides a substrate for longer-term integration, or even storage, of information about the fly's body rotations. During a multistep, non-stereotyped behavior like takeoff, modulation of responses to visual stimuli by haltere input might help the fly's brain transition from a standing state (where the halteres are stationary and not providing any mechanosensation) to flight. Additionally, the CX could distinguish self-motion (potentially using efference copy) from passive stimulation (as used here and by Homberg, 1994), or assess haltere symmetry. Longer-term integration of haltere input may be useful (though almost certainly not sufficient) for behaviors like path integration (Kim and Dickinson, 2017), allowing the fly to integrate the position of its body over time. In mammalian brains, vestibular information is critical for directional and spatial representations in the hippocampus for navigation (Stackman et al., 2002). This may also be the case in flies, where heading-encoding cells of the CX can maintain a heading representation without visual cues (Seelig and Jayaraman, 2015). This would suggest the CX is a good candidate for the integration of visual and nonvisual sensory modalities used in such tasks as path integration. When visual cues are not available or are unreliable, haltere inputs could provide information necessary for these higher-order behaviors.

\section{References}

Batschelet E (1981) Circular statistics in biology. New York: Academic.

Burnham KP, Anderson DR (1998) Model selection and inference: a practical information-theoretical approach. New York: Springer.

Burnham KP, Anderson DR (2004) Multimodel inference: understanding AIC and BIC in model selection. Sociol Methods Res 33:261-304.

Chan WP, Dickinson MH (1996) Position-specific central projections of mechanosensory neurons on the haltere of the blow fly, Calliphora vicina. J Comp Neurol 369:405-418.

Chen G, King JA, Burgess N, O'Keefe J (2013) How vision and movement combine in the hippocampal place code. Proc Natl Acad Sci U S A 110: 378-383.

Chiappe ME, Seelig JD, Reiser MB, Jayaraman V (2010) Walking modulates speed sensitivity in Drosophila motion vision. Curr Biol 20:1470-1475.

Deora T, Singh AK, Sane SP (2015) Biomechanical basis of wing and haltere coordination in flies. Proc Natl Acad Sci U S A 112:1481-1486.

Dickinson MH (1999) Haltere-mediated equilibrium reflexes of the fruit fly, Drosophila melanogaster. Philos Trans R Soc Lond B Biol Sci 354:903-916.

Dickinson MH, Muijres FT (2016) The aerodynamics and control of free flight manoeuvres in Drosophila. Philos Trans R Soc Lond B Biol Sci 371:20150388.

Duistermars BJ, Chow DM, Condro M, Frye MA (2007) The spatial, temporal and contrast properties of expansion and rotation flight optomotor responses in Drosophila. J Exp Biol 210:3218-3227.

el Jundi B, Pfeiffer K, Heinze S, Homberg U (2014) Integration of polarization and chromatic cues in the insect sky compass. J Comp Physiol A Neuroethol Sens Neural Behav Physiol 200:575-589.

el Jundi B, Warrant EJ, Byrne MJ, Khaldy L, Baird E, Smolka J, Dacke M (2015) Neural coding underlying the cue preference for celestial orientation. Proc Natl Acad Sci U S A 112:11395-11400.

Fayyazuddin A, Dickinson MH (1996) Haltere afferents provide direct, electrotonic input to a steering motor neuron in the blowfly, Calliphora. J Neurosci 16:5225-5232.

Fayyazuddin A, Dickinson MH (1999) Convergent mechanosensory input structures the firing phase of a steering motor neuron in the blowfly, Calliphora. J Neurophysiol 82:1916-1926.

Fedele G, Edwards MD, Bhutani S, Hares JM, Murbach M, Green EW, Dissel S, Hastings MH, Rosato E, Kyriacou CP (2014) Genetic analysis of circadian responses to low frequency electromagnetic fields in Drosophila melanogaster. PLoS Genet 10:e1004804.

Fox JL, Daniel TL (2008) A neural basis for gyroscopic force measurement in the halteres of holorusia. J Comp Physiol A Neuroethol Sens Neural Behav Physiol 194:887-897.

Fox JL, Fairhall AL, Daniel TL (2010) Encoding properties of haltere neurons enable motion feature detection in a biological gyroscope. Proc Natl Acad Sci U S A 107:3840-3845.

Frye MA, Dickinson MH (2004) Closing the loop between neurobiology and flight behavior in Drosophila. Curr Opin Neurobiol 14:729-736.

Gao X, Wehr M (2015) A coding transformation for temporally structured sounds within auditory neurons. Neuron 86:292-303.

Gegear RJ, Casselman A, Waddell S, Reppert SM (2008) Cryptochrome mediates light-dependent megnetosensitivity in Drosophila. Nature 454:10141018.

Green J, Adachi A, Shah KK, Hirokawa JD, Magani PS, Maimon G (2017) A neural circuit architecture for angular integration in Drosophila. Nature 546:101-106.

Haag J, Wertz A, Borst A (2010) Central gating of fly optomotor response. Proc Natl Acad Sci U S A 107:20104-20109.

Hall JM, McLoughlin DP, Kathman ND, Yarger AM, Mureli S, Fox JL (2015) Kinematic diversity suggests expanded roles for fly halteres. Biol Lett 11:20150845.

Heide G (1983) Neural mechanisms of flight control in Diptera. In: BIONA report, Ed 2 (Akademie der Wissenschaften und der Literatur zu Mainz and Gustav Fischer Verlag, Mainz) (Nachtigall W, ed), pp 35-52. Stuttgart: G. Fischer.

Heinze S, Homberg U (2007) Maplike representation of celestial E-vector orientations in the brain of an insect. Science 315:995-997.

Heinze S, Reppert SM (2011) Sun compass integration of skylight cues in migratory monarch butterflies. Neuron 69:345-358.

Hengstenberg R (1988) Mechanosensory control of compensatory head roll during flight in the blowfly Calliphora erythrocephala Meig. J Comp Physiol A 163:151-165.

Hengstenberg R (1993) Multisensory control in insect oculomotor systems. In: Vision motion and its role in the stabilization of gaze (Miles F, Wallman J, eds), pp 285-298. New York: Elsevier.

Homberg U (1994) Flight-correlated activity changes in neurons of the lateral accessory lobes in the brain of the locust Schistocerca gregaria. J Comp Physiol A 175:597-610.

Homberg U, Heinze S, Pfeiffer K, Kinoshita M, el Jundi B (2011) Central neural coding of sky polarization in insects. Philos Trans R Soc Lond B Biol Sci 366:680-687.

Huston SJ, Krapp HG (2009) Nonlinear integration of visual and haltere inputs in fly neck motor neurons. J Neurosci 29:13097-13105.

Jacob PY, Poucet B, Liberge M, Save E, Sargolini F (2014) Vestibular control of entorhinal cortex activity in spatial navigation. Front Integr Neurosci $8: 38$.

Kathman ND, Kesavan M, Ritzmann RE (2014) Encoding wide-field mo- 
tion and direction in the central complex of the cockroach Blaberus discoidalis. J Exp Biol 217:4079-4090.

Kim IS, Dickinson MH (2017) Idiothetic path integration in the fruit fly Drosophila melanogaster. Curr Biol 27:2227-2238.e3.

Land MF, Collett TS (1974) Chasing behaviour of houseflies (Fannia canicularis). J Comp Physiol 89:331-357.

Lanman J, Bizzi E, Allum J (1978) The coordination of eye and head movement during smooth pursuit. Brain Res 153:39-53.

Maimon G, Straw AD, Dickinson MH (2010) Active flight increases the gain of visual motion processing in Drosophila. Nat Neurosci 13:393-399.

Martin JP, Guo P, Mu L, Harley CM, Ritzmann RE (2015) Central-complex control of movement in the freely walking cockroach. Curr Biol 25:2795-2803.

Mureli S, Fox JL (2015) Haltere mechanosensory influence on tethered flight behavior in Drosophila. J Exp Biol 218:2528-2537.

Mureli S, Thanigaivelan I, Schaffer ML, Fox JL (2017) Cross-modal influence of mechanosensory input on gaze responses to visual motion in Drosophila. J Exp Biol 220:2218-2227.

Nalbach G (1993) The halteres of the blowfly Calliphora 1. Kinematics and dynamics. J Comp Physiol 173:293-300.

Namiki S, Kanzaki R (2016) Comparative neuroanatomy of the lateral accessory lobe in the insect brain. Front Physiol 7:244.

Nordmann GC, Hochstoeger T, Keays DA (2017) Magnetoreception: a sense without a receptor. PLoS Biol 15:e2003234.

Pegel U, Pfeiffer K, Homberg U (2018) Integration of celestial compass cues in the central complex of the locust brain. J Exp Biol 221:jeb171207.

Pfeiffer K, Kinoshita M, Homberg U (2005) Polarization-sensitive and light-sensitive neurons in two parallel pathways passing through the anterior optic tubercle in the locust brain. J Neurophysiol 94:3903-3915.

Phillips-Portillo J (2012) The central complex of the flesh fly, Neobellieria bullata: recordings and morphologies of protocerebral inputs and smallfield neurons. J Comp Neurol 520:3088-3104.

Pringle JWS (1948) The gyroscopic mechanism of the halteres of diptera. Philos Trans R Soc B Biol Sci 233:347-384.

Reiser MB, Dickinson MH (2008) A modular display system for insect behavioral neuroscience. J Neurosci Methods 167:127-139.

Ritzmann RE, Ridgel AL, Pollack AJ (2008) Multi-unit recording of antennal mechano-sensitive units in the central complex of the cockroach, Blaberus discoidalis. J Comp Physiol A Neuroethol Sens Neural Behav Physiol 194:341-360.

Seelig JD, Jayaraman V (2013) Feature detection and orientation tuning in the Drosophila central complex. Nature 503:262-266.

Seelig JD, Jayaraman V (2015) Neural dynamics for landmark orientation and angular path integration. Nature 521:186-191.

Srinivasan MV (2000) Honeybee navigation: nature and calibration of the “odometer." Science 287:851-853.
Srinivasan MV (2015) Where paths meet and cross: navigation by path integration in the desert ant and the honeybee. J Comp Physiol A Neuroethol Sens Neural Behav Physiol 201:533-546.

Stackman RW, Clark AS, Taube JS (2002) Hippocampal spatial representations require vestibular input. Hippocampus 12:291-303.

Stone T, Webb B, Adden A, Weddig NB, Honkanen A, Scimeca L, Warrant E, Heinze S, Stone T, Webb B, Adden A, Templin R, Wcislo W, Scimeca L, Warrant E, Heinze S (2017) An anatomically constrained model for path integration in the bee brain. Curr Biol 27:3069-3085.e11.

Strausfeld NJ (2012) Arthropod brains: evolution, functional elegance, and historical significance. Cambridge, MA: Harvard UP.

Strausfeld NJ, Seyan HS (1985) Convergence of visual, haltere, and prosternal inputs at neck motor neurons of calliphora erythrocephala. Cell Tissue Res 240:601-615.

Strauss R, Berg C (2010) The central control of oriented locomotion in insects: towards a neurobiological model. The 2010 International Joint Conference on Neural Networks (IJCNN), Barcelona pp 1-8.

Suver MP, Mamiya A, Dickinson MH (2012) Octopamine neurons mediate flight-induced modulation of visual processing in Drosophila. Curr Biol 22:2294-2302.

Theobald JC, Ringach DL, Frye MA (2010) Dynamics of optomotor responses in Drosophila to perturbations in optic flow. J Exp Biol 213: $1366-1375$

Thompson RA, Wehling MF, Evers JH, Dixon WE (2009) Body rate decoupling using haltere mid-stroke measurements for inertial flight stabilization in Diptera. J Comp Physiol A 195:99-112.

Tryba AK, Ritzmann RE (2000) Multi-joint coordination during walking and foothold searching in the blaberus cockroach: II. Extensor motor neuron pattern. J Neurophysiol 83:3337-3350.

Turner-Evans D, Wegener S, Rouault H, Franconville R, Wolff T, Seelig JD, Druckmann S, Jayaraman V (2017) Angular velocity integration in a fly heading circuit. Elife 6:e23496.

Varga AG, Ritzmann RE (2016) Cellular basis of head direction and contextual cues in the insect brain. Curr Biol 26:1816-1828.

Wehner R, Michel B, Antonsen P (1996) Visual navigation in insects: coupling of egocentric and geocentric information. J Exp Biol 199:129-140.

Weir PT, Schnell B, Dickinson MH (2014) Central complex neurons exhibit behaviorally gated responses to visual motion in Drosophila. J Neurophysiol 111:62-71.

Yarger AM, Fox JL (2016) Dipteran halteres: perspectives on function and integration for a unique sensory organ. Integr Comp Biol 56:865-876.

Yarger AM, Fox JL (2018) Single mechanosensory neurons encode lateral displacements using precise spike timing and thresholds. Proc Biol Sci 285:20181759. 